\title{
Photon-jet correlations in hadronic collisions
}

\section{Tomasz Pietrycki*}

Institute of Nuclear Physics, PL-31-342 Cracow, Poland

E-mail: Tomasz.Pietrycki@ifj.edu.pl

\section{Antoni Szczurek}

Institute of Nuclear Physics, PL-31-342 Cracow, Poland

and University of Rzeszów, PL-35-959 Rzeszów, Poland

E-mail: Antoni.Szczurek@ifj.edu.pl

We compare results of $k_{t}$-factorization approach and next-to-leading order collinear-factorization approach for photon-jet correlations in $p p$ and $p \bar{p}$ collisions at RHIC, Tevatron and LHC energies. We discuss correlations in azimuthal angle as well as in two-dimensional space of transverse momenta of photon and jet. Different unintegrated parton distributions (UPDF) are included in the $k_{t}$-factorization approach. The results depend on UPDFs used. The results of NLO collinearfactorization are shown for comparison.

High-pT physics at LHC

March 23-27 2007

University of Jyväskylä, Jyväskylä, Finland

* Speaker. 


\section{Introduction}

The jet-jet correlations are interesting probe of QCD dynamics [1]. Recent studies of hadronhadron correlations at RHIC [2] open a new possibility to study the dynamics of jet and particle production. The hadron-hadron correlations involve both jet-jet correlations as well as complicated jet structure. Recently also preliminary data on photon-hadron azimuthal correlation in nuclear collisions were presented [3]. In principle, such correlations should be easier for theoretical description as here only one jet enters, at least in leading order pQCD. On the experimental side, such measurements are more difficult due to much reduced statistics compared to the dijet studies.

Up to now no theoretical calculation for photon-jet were presented in the literature, even for elementary collisions. In leading-order collinear-factorization approach the photon and the associated jet are produced back-to-back. If transverse momenta of partons entering the hard process are included, the transverse momenta of the photon and the jet are no longer balanced and finite (non-zero) correlations in a broad range of relative azimuthal angle and/or in lengths of transverse momenta of the photon and the jet are obtained. The finite correlations can be also obtained in higher-order collinear-factorization approach [4]. According to our knowledge no detailed studies of photon-jet correlations were presented in the literature. Only cross section as a function of photon-jet invariant mass was discussed for polarized pp collisions [5].

\section{Formalism}

It is known that at midrapidities and at relatively small transverse momenta the photon-jet production is dominated by (sub)processes initiated by gluons. Here we concentrate on direct photons. A large fraction of the photons could come from the $g g \rightarrow g g$ process, where one gluon fragments into the photon and the other produces the jet. In principle, such mechanisms can be removed by imposing isolation cuts.

In the $k_{t}$-factorization approach the cross section for a simultaneous production of a photon and an associated jet in the collisions of two hadrons ( $p p$ or $p \bar{p})$ can be written as

$$
\begin{aligned}
\frac{d \sigma_{h_{1} h_{2} \rightarrow \gamma k}}{d^{2} p_{1, t} d^{2} p_{2, t}}=\int d y_{1} d y_{2} \frac{d^{2} k_{1, t}}{\pi} \frac{d^{2} k_{1, t}}{\pi} \frac{1}{16 \pi^{2}\left(x_{1} x_{2} s\right)^{2}} \overline{\left|\mathscr{M}_{i j \rightarrow \gamma k}\right|^{2}} \\
\cdot \delta^{2}\left(\vec{k}_{1, t}+\vec{k}_{2, t}-\vec{p}_{1, t}-\vec{p}_{2, t}\right) \mathscr{F}_{i}\left(x_{1}, k_{1, t}^{2}, \mu_{1}^{2}\right) \mathscr{F}_{j}\left(x_{2}, k_{2, t}^{2}, \mu_{2}^{2}\right),
\end{aligned}
$$

where $\mathscr{F}_{i}\left(x_{1}, k_{1, t}^{2}, \mu_{1}^{2}\right)$ and $\mathscr{F}_{j}\left(x_{2}, k_{2, t}^{2}, \mu_{2}^{2}\right)$ are so-called unintegrated parton distributions. The longitudinal momentum fractions are evaluated as $x_{1}=\left(m_{1 t} \mathrm{e}^{+y_{1}}+m_{2 t} \mathrm{e}^{+y_{2}}\right) / \sqrt{s}$ and $x_{2}=\left(m_{1 t} \mathrm{e}^{-y_{1}}+\right.$ $\left.m_{2 t} \mathrm{e}^{-y_{2}}\right) / \sqrt{s}$. The final partonic state is $\gamma k=\gamma g, \gamma q$. If one makes the following replacement $\mathscr{F}_{i}\left(x_{1}, k_{1, t}^{2}\right) \rightarrow x_{1} p_{i}\left(x_{1}\right) \boldsymbol{\delta}\left(k_{1, t}^{2}\right), \mathscr{F}_{j}\left(x_{2}, k_{2, t}^{2}\right) \rightarrow x_{2} p_{j}\left(x_{2}\right) \boldsymbol{\delta}\left(k_{2, t}^{2}\right)$ and $\mathscr{M}_{i j \rightarrow \gamma k}\left(k_{1, t}^{2}, k_{2, t}^{2}\right) \rightarrow \mathscr{M}_{i j \rightarrow \gamma k}\left(k_{1, t}^{2}=\right.$ $\left.0, k_{2, t}^{2}=0\right)$ then one recovers the standard leading-order collinear formula.

Up to now we have concentrated only on processes with two explicit hard partons $(\gamma k)$ in the $k_{t}$-factorization approach. It is of interest to compare results of our approach with the standard collinear next-to-leading order approach.

The cross section for $h_{1} h_{2} \rightarrow \gamma k l X$ processes can be calculated according to the standard parton model formula

$$
d \sigma_{h_{1} h_{2} \rightarrow \gamma k l}=\sum_{i j k l} \int d x_{1} d x_{2} p_{i}\left(x_{1}, \mu^{2}\right) p_{j}\left(x_{2}, \mu^{2}\right) d \hat{\sigma}_{i j \rightarrow \gamma k l}
$$


where elementary cross section can be written as

$$
d \hat{\sigma}_{i j \rightarrow \gamma k l}=\frac{1}{2 \hat{s}} \overline{\left|\mathscr{M}_{i j \rightarrow \gamma k l}\right|^{2}}(2 \pi)^{4} \delta^{4}\left(p_{a}+p_{b}-\sum_{i=1}^{3} p_{i}\right) \prod_{i=1}^{3} \frac{d^{3} p_{i}}{2 E_{i}(2 \pi)^{3}} .
$$

Repeating similar steps as for $2 \rightarrow 2$ processes we get finally

$$
\begin{aligned}
d \hat{\sigma}_{i j \rightarrow \gamma k l} & =\sum_{i j k l} \frac{1}{64 \pi^{4} \hat{s}^{2}} x_{1} p_{i}\left(x_{1}, \mu^{2}\right) x_{2} p_{j}\left(x_{2}, \mu^{2}\right) \overline{\left|\mathscr{M}_{i j \rightarrow \gamma k l}\right|^{2}} \\
& \times p_{1, t} d p_{1, t} p_{2, t} d p_{2, t} d \phi_{-} d y_{1} d y_{2} d y_{3}
\end{aligned}
$$

where the relative azimuthal angle between the photon and associated jet $\phi_{-}$is restricted to the interval $(0, \pi)$. The last formula is very useful in calculating the cross section for particle 1 and particle 2 correlations.

\section{Results}

In this section we shall present results for RHIC, Tevatron and LHC energies. We use UPDFs from the literature. There are only two complete sets of UPDFs in the literature which include not only gluon distributions but also distributions of quarks and antiquarks: (a) Kwieciński [7], (b) Kimber-Martin-Ryskin [8].

For comparison we shall include also unintegrated distributions obtained from collinear ones by the Gaussian smearing procedure. Such a procedure is often used in the context of inclusive direct photon production $[9,10]$. Comparing results obtained with those Gaussian distributions and the results obtained with the Kwieciński distributions with nonperturbative Gaussian form factors will allow to quantify the effect of UPDF evolution as contained in the Kwieciński evolution equations. What is the hard scale for our process? In our case the best candidate for the scale is the photon and/or jet transverse momentum. Since we are interested in rather small transverse momenta the evolution length is not too large and the deviations from initial $k_{t}$-distributions (assumed here to be Gaussian) should not be too big.

At high energies one enters into a small-x region, i.e. the region of a specific dynamics of the QCD emissions. In this region only unintegrated distributions of gluons exist in the literature. In our case the dominant contributions come from QCD-Compton gluon-quark or quark - gluon initiated hard subprocesses. This means that we need unintegrated distributions of both gluons and quarks/antiquarks. In this case we take such UGDFs from the literature and supplement them by the Gaussian distributions of quarks/antiquarks.

Let us start from presenting our results on the $\left(p_{1, t}, p_{2, t}\right)$ plane. In Fig.1 we show the maps for different UPDFs used in the $k_{t}$-factorization approach as well as for NLO collinear-factorization approach for $p_{1, t}, p_{2, t} \in(5,20) \mathrm{GeV}$ and at the Tevatron energy $W=1960 \mathrm{GeV}$. In the case of the Kwieciński distribution we have taken $b_{0}=1 \mathrm{GeV}^{-1}$ for the exponential nonperturbative form factor and the scale parameter $\mu^{2}=100 \mathrm{GeV}^{2}$. Rather similar distributions are obtained for different UPDFs. The distribution obtained in the collinear NLO approach differs qualitatively from those obtained in the $k_{t}$-factorization approach. First of all, one can see a sharp ridge along the diagonal $p_{1, t}=p_{2, t}$. This ridge corresponds to a soft singularity when the unobserved parton has a very small 
transverse momentum $p_{3, t}$. As will be clear in a moment this corresponds to the azimuthal angle between the photon and the jet being $\phi_{-}=\pi$. Obviously this is a region which cannot be reliably calculated in collinear $\mathrm{pQCD}$. There are different practical possibilities to exclude this region from the calculations. The most primitive way (possible only in theoretical calculations) is to impose a lower cut on transverse momentum of the unobserved parton $p_{3, t}$. Secondly, the standard collinear NLO approach generates much bigger cross section for asymmetric $p_{1, t}$ and $p_{2, t}$.
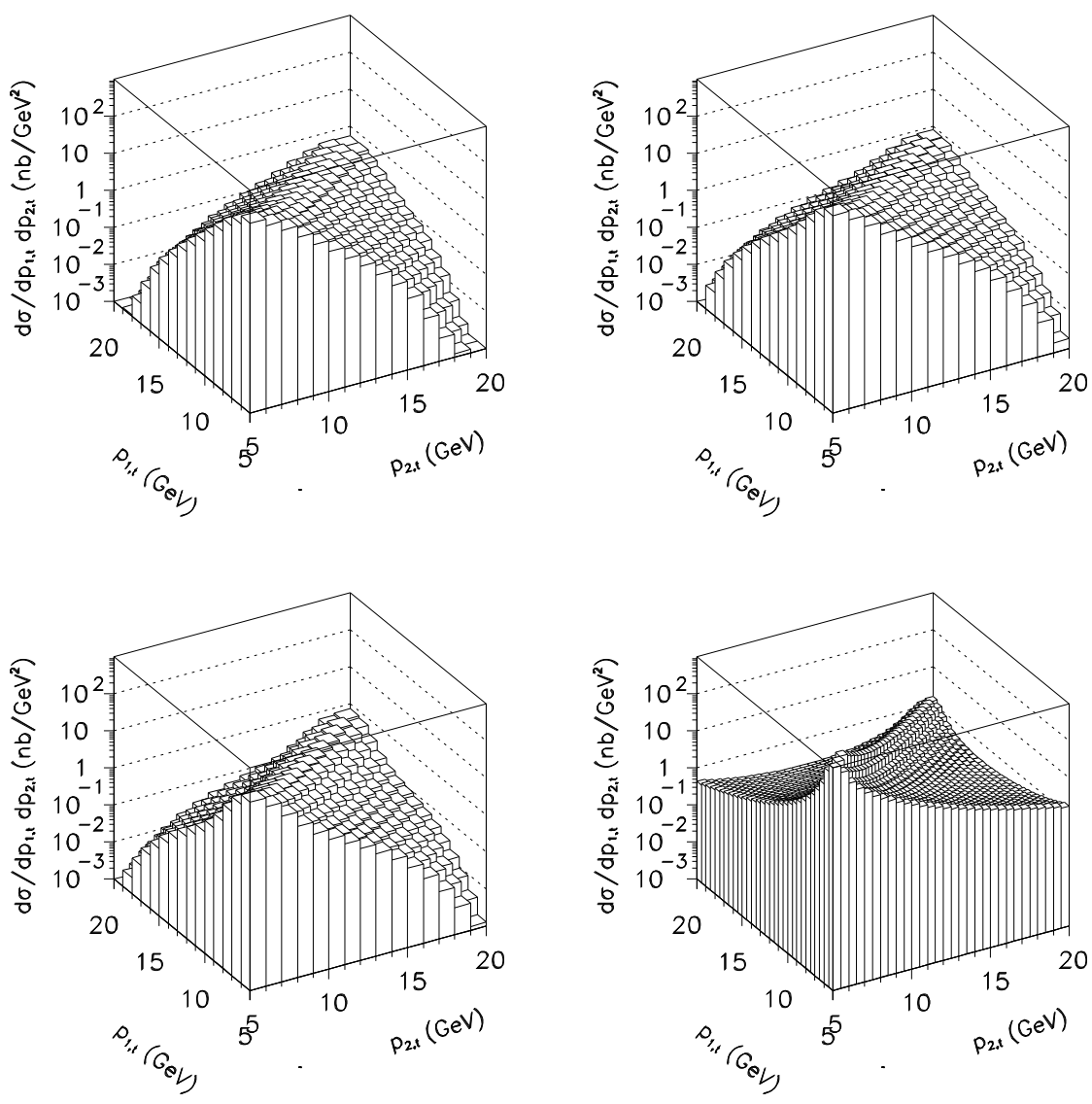

Figure 1: Transverse momentum distributions $d \sigma / d p_{1, t} d p_{2, t}$ at $\mathrm{W}=1960 \mathrm{GeV}$ and for different UPDFs in the $k_{t}$-factorization approach for Kwieciński $\left(b_{0}=1 \mathrm{GeV}^{-1}, \mu^{2}=100 \mathrm{GeV}^{2}\right.$ ) (a), BFKL (b), KL (c) and NLO $2 \rightarrow 3$ collinear-factorization approach including diagrams from Fig.2. in Ref.[11] (d). The integration over rapidities from the interval $-5<y_{1}, y_{2}<5$ is performed.

As discussed in Ref.[6] the Kwieciński unintegrated parton distributions are very useful to treat both the nonperturbative (intrinsic nonperturbative transverse momenta) and the perturbative (QCD broadening due to parton emission) effects on the same footing. In Fig.2 we show the effect of the scale evolution of the Kwieciński UPDFs on the azimuthal angle correlations between the photon and the associated jet. We show results for different initial conditions $\left(b_{0}=0.5,1.0,2.0\right.$ $\mathrm{GeV}^{-1}$ ). At the initial scale (fixed here as in the original GRV [12] to be $\mu^{2}=0.25 \mathrm{GeV}^{2}$ ) there is a sizeable difference of the results for different $b_{0}$. The difference becomes less and less pronounced when the scale increases. At $\mu^{2}=100 \mathrm{GeV}^{2}$ the differences practically disappear. This is due to 
the fact that the QCD-evolution broadening of the initial parton transverse momentum distribution is much bigger than the typical initial nonperturbative transverse momentum scale.
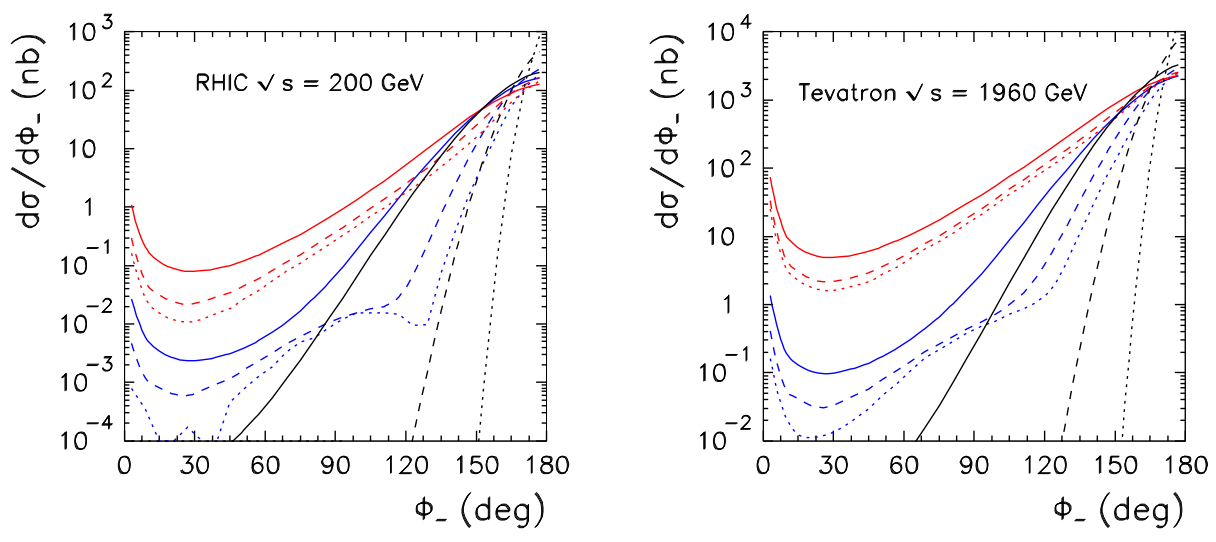

Figure 2: (Color on line) Azimuthal angle correlation functions at (a) RHIC, (b) Tevatron energies for different scales and different values of $b_{0}$ of the Kwieciński distributions. The solid line is for $b_{0}=0.5 \mathrm{GeV}^{-1}$, the dashed line is for $b_{0}=1 \mathrm{GeV}^{-1}$ and the dotted line is for $b_{0}=2 \mathrm{GeV}^{-1}$. Three different values of the scale parameters are shown: $\mu^{2}=0.25,10,100 \mathrm{GeV}^{2}$ (the bigger the scale the bigger the decorellation effect, different colors on line). In this calculation $p_{1, t}, p_{2, t} \in(5,20) \mathrm{GeV}$ and $y_{1}, y_{2} \in(-5,5)$.

In Fig. 3 we show corresponding azimuthal angular correlations for three different energies relevant for RHIC, Tevatron and LHC. In this case integration is made over transverse momenta $p_{1, t}, p_{2, t} \in(5,20) \mathrm{GeV}$ and rapidities $y_{1}, y_{2} \in(-5,5)$. The standard NLO collinear cross section grows somewhat faster with energy than the $k_{t}$-result with unintegrated Kwieciński distribution. This is partially due to approximation made in calculation of the off-shell matrix elements. Up to now we have used matrix elements called "on-shell" (for explanation see appendix A in Ref.[11]). This approximation is expected to be reliable for small transverse momenta of gluons (for a detailed discussion see Ref.[6]). For larger gluon transverse momenta the longitudinal gluons start to play important role. This is obiously not included in our simple extrapolation of the on-shell formula.

In Fig.4 we show angular azimuthal correlations for different relations between transverse momenta of outgoing photon and partons: (a) with no constraints on $p_{3, t}$, (b) the case where $p_{2, t}>$ $p_{3, t}$ condition (called leading jet condition in the following) is imposed, (c) $p_{2, t}>p_{3, t}$ and an additional condition $p_{1, t}>p_{3, t}$. The results depend significantly on the scenario chosen as can be seen from the figure. The general pattern is very much the same for different energies.

Fig.4 suggests that the $k_{t}$-factorization approach may be very efficient to describe correlation in azimuth for $\phi_{-}<\pi / 2$ (where NLO contribution vanishes) when the leading jet condition is imposed.

\section{Conclusions}

We have performed for the first time the lacking in the literature calculation of the photon-jet correlation observables in proton-(anti)proton (RHIC, Tevatron and LHC) collisions. Up to now 

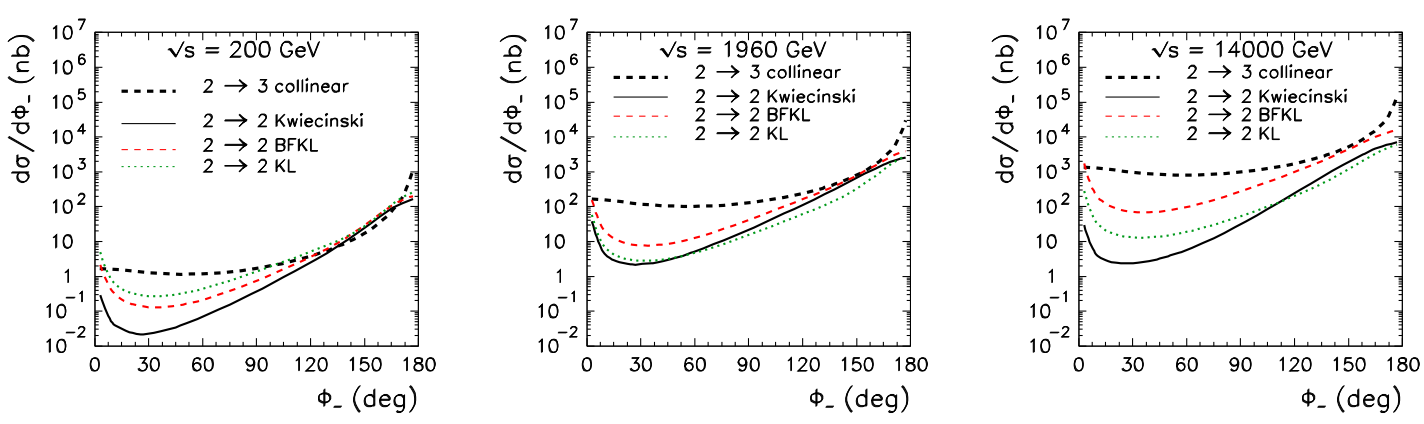

Figure 3: Photon-jet angular azimuthal correlations $d \sigma / d \phi_{-}$for proton-(anti)proton collision at $W=$ $200,1960,14000 \mathrm{GeV}$ for different UPDFs in the $k_{t}$-factorization approach for the Kwieciński (solid), BFKL (dashed), KL (dotted) UPDFs/UGDFs and for the NLO collinear-factorization approach (thick dashed). Here $y_{1}, y_{2} \in(-5,5)$.
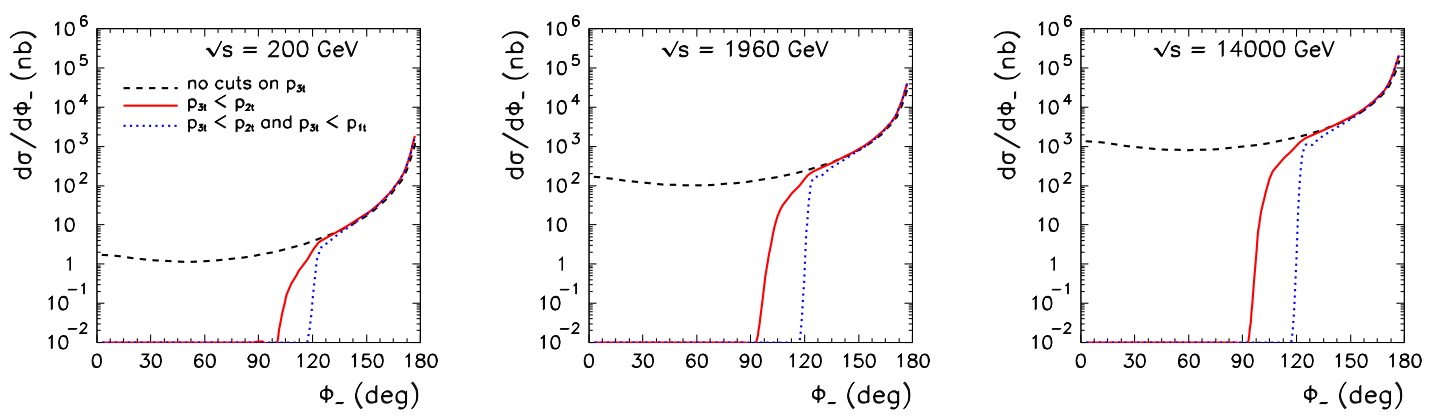

Figure 4: Angular azimuthal correlations in the NLO collinear-factorization approach without any extra constraints (dashed), $p_{3, t}<p_{2, t}$ (solid), $p_{3, t}<p_{2, t}$ and $p_{3, t}<p_{1, t}$ in addition (dotted). Here $W=$ $200,1960,14000 \mathrm{GeV}$ and $y_{1}, y_{2} \in(-5,5)$.

such correlations have not been studied experimentally either. We have concentrated on the region of small transverse momenta (semi-hard region) where the $k_{t}$-factorization approach seems to be the most efficient and theoretically justified tool. We have calculated correlation observables for different unintegrated parton distributions from the literature. Our previous analysis of inclusive spectra of direct photons suggests that the Kwieciński distributions give the best description of existing experimental data at low and intermediate energies.

The correlation function depends strongly on whether it is the correlation of the photon and any jet or the correlation of the photon and the leading jet. In the last case there are regions in azimuth and/or in the two-dimensional $\left(p_{1, t}, p_{2, t}\right)$ space which cannot be populated in the standard next-to-leading order approach. In the latter case the $k_{t}$-factorization seems to be a useful and efficient tool.

We hope that the photon-jet correlations will be measured at Tevatron. At RHIC one can measure jet-hadron correlations for rather not too high transverse momenta of the trigger photon and of the associated hadron. This is precisely the semihard region discussed here. In this case 
the theoretical calculations would require inclusion of the fragmentation process. This can be done easily assuming independent parton fragmentation method using fragmentation functions extracted from $e^{+} e^{-}$collisions. This analysis is in progress.

Acknowledgments We are indebted to Jan Rak from the PHENIX collaboration for the discussion of recent results for photon-hadron correlations at RHIC. This work was partially supported by the grant of the Polish Ministry of Scientific Research and Information Technology number 1 P03B 02828.

\section{References}

[1] S.S. Adler et al. (PHENIX collaboration), Phys. Rev. Lett. 97 (2006) 052301;

S.S. Adler et al. (PHENIX collaboration), Phys. Rev. C73 (2006) 054903;

S.S. Adler et al. (PHENIX collaboration), Phys. Rev. Lett. 96 (2006) 222301;

M. Oldenburg et al. (STAR collaboration), Nucl. Phys. A774 (2006) 507.

[2] S.S. Adler et al. (PHENIX collaborations), Phys. Rev. D74 (2006) 072002.

[3] DongJo Kim, a talk at the international workshop on "High- $p_{t}$ processes at LHC", Jyväskylä, Finland, March 23-28, 2007.

[4] F.A. Berends, R. Kleiss, P.De Causmaecker, R. Gastmans and T.T. Wu, Phys. Lett. B 103124 (1981).

[5] S. Frixione and W. Vogelsang, Nucl. Phys. B 568 (2000) 60.

[6] T. Pietrycki and A. Szczurek, Phys. Rev. D75 014023 (2007).

[7] J. Kwieciński, Acta Phys. Polon. B33 1809 (2002);

A. Gawron and J. Kwieciński, Acta Phys. Polon. B34 133 (2003);

A. Gawron, J. Kwieciński and W. Broniowski, Phys. Rev. D68 054001 (2003).

[8] M.A. Kimber, A.D. Martin and M.G. Ryskin, Phys. Rev. D63 114027 (2001).

[9] J.F. Owens, Rev. Mod. Phys. 59465 (1987).

[10] U. d'Alesio and F. Murgia, Phys. Rev. D70 074009 (2004).

[11] T. Pietrycki, A. Szczurek, [arXiv:0704.2158].

[12] M. Glück, E. Reya and A. Vogt, Eur. Phys. J. C5 461 (1998). 